\title{
Natural convection in porous media under cross temperature and concentration gradients with Boundary Element Method
}

\author{
J. Kramer ${ }^{1}$, R. Jecl ${ }^{1}$ \& L. Š Skerget ${ }^{2}$ \\ ${ }^{1}$ Faculty of Civil Engineering, University of Maribor, Slovenia \\ ${ }^{2}$ Faculty of Mechanical Engineering, University of Maribor, Slovenia
}

\begin{abstract}
Double-diffusive natural convection in a horizontal enclosure is studied numerically with the use of Boundary Element Method. The enclosure is heated and cooled along vertical walls, while horizontal walls are maintained at different concentration values. The Brinkman model is used to simulate the fluid flow through porous media, the density variation is taken into account by the Boussinesq approximation. The governing parameters of the problem are the Darcy number, porous Rayleigh number, Lewis number and buoyancy coefficient. Different flow regimes for thermal and solutal dominated flows are demonstrated for different values of governing parameters. The results are compared to those in published studies, obtained with other approximative methods.

Keywords: Boundary Element Method, porous medium, double-diffusive natural convection, Brinkman model.
\end{abstract}

\section{Introduction}

Double-diffusive natural convection in a saturated porous media is of fundamental importance in many engineering branches e.g. civil engineering, mechanical engineering, chemical engineering. Several problems in nature or industry can be modeled as convective flow in enclosure filled with porous media such as underground spreading of pollutants, geophysical systems, fuel storage installations. In general two main configurations have been considered in the literature: heat and mass gradients are imposed horizontally along the enclosure or heat and mass gradients are imposed vertically along the height of the enclosure. Only few recently 


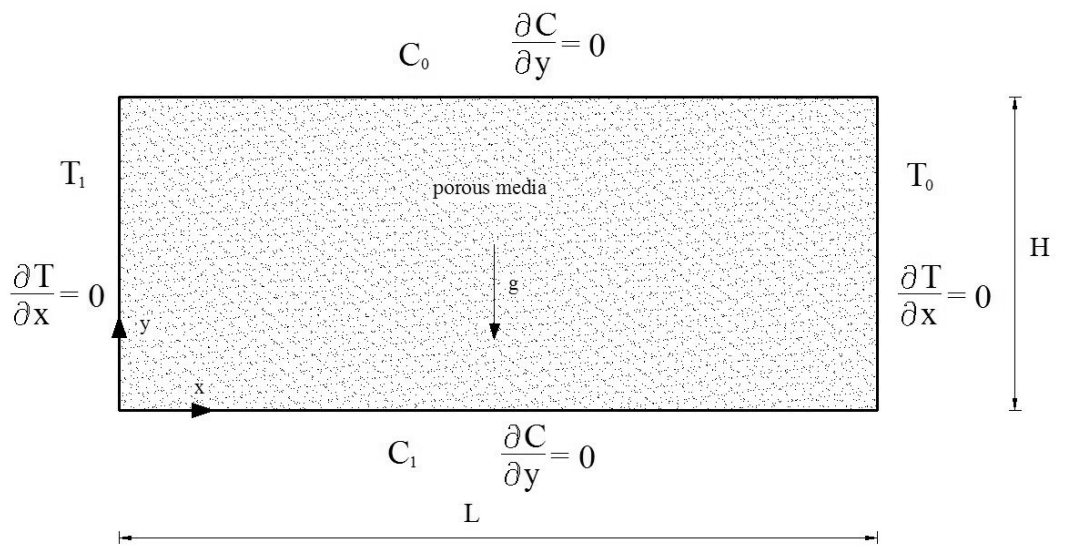

Figure 1: Geometry with boundary conditions for convection with horizontal temperature and vertical solutal gradient.

published studies are dealing cross relation between the temperature and solutal gradient (temperature or concentration gradient imposed horizontally and concentration or temperature gradient imposed vertically) [1-3]. In the studies is shown, that the competition between the thermal and solutal force produce very complex flow patterns.

In the present work the numerical results for heat and solute transfer in a horizontal cavity of aspect ratio $A=2$, subjected to cross temperature and solute gradients are presented. In the analysis the Brinkman model is adopted while the governing set of equations is solved with use of the extended Boundary Element Method. The results are obtained for different governing parameters, the accuracy of proposed numerical scheme is stated with comparison to some published results.

\section{Mathematical model}

The physical model with corresponding boundary conditions is shown in Figure 1. The problem under consideration is a two dimensional enclosure with aspect ratio $A=L / H=2$ filled with fully saturated porous media. The vertical walls are subjected to temperature differences ( $T_{1}$ and $T_{0}$ respectively) while the horizontal walls are subjected to fixed concentrations (high concentration at the bottom $C_{1}$ and low concentration at the top $C_{0}$ ). The main assumptions are that the flow is incompressible, steady, laminar, the fluid is Newtonian. The solid matrix is nondeformable, homogenous, isotropic and in thermal equilibrium with the fluid phase.

Transport phenomena in porous media is mathematically described with conservation equations for mass, momentum, energy and species, which are written at the macroscopic level considering the fact that only a part of the volume, expressed with porosity $\phi$, is available for the fluid flow [4]. The general set of equations is 
given as:

- Continuity:

$$
\frac{\partial v_{i}}{\partial x_{i}}=0
$$

- Momentum:

$$
\frac{1}{\phi} \frac{\partial v_{i}}{\partial t}+\frac{1}{\phi^{2}} \frac{\partial v_{j} v_{i}}{\partial x_{j}}=-\frac{1}{\rho_{0}} \frac{\partial p}{\partial x_{i}}+F g_{i}-\frac{v}{K} v_{i}+\frac{\partial}{\partial x_{j}}\left(2 \frac{v}{\phi} \dot{\varepsilon}_{i j}\right),
$$

- Energy:

$$
\frac{\partial}{\partial t}\left[\phi c_{f}+(1-\phi) c_{s}\right] T+c_{f} \frac{\partial v_{j} T}{\partial x_{j}}=\frac{\partial}{\partial x_{j}}\left(\lambda_{e} \frac{\partial T}{\partial x_{j}}\right),
$$

- Species:

$$
\phi \frac{\partial C}{\partial t}+\frac{\partial v_{j} C}{\partial x_{j}}=\frac{\partial}{\partial x_{j}}\left(D \frac{\partial C}{\partial x_{j}}\right) .
$$

The parameters, used above are: $v_{i}$ volume-averaged velocity, $x_{i}$ the i-th coordinate, $\phi$ porosity, $t$ time, $\rho$ density, $v$ kinematic viscosity, $\partial p / \partial x_{i}$ the pressure gradient, $g_{i}$ gravity and $K$ permeability of porous media. Parameters $c_{f}=(\rho c)_{f}$ and $c_{s}=(\rho c)_{s}$ are the heat capacities for the fluid and solid phases, respectively, $T$ is temperature, $\lambda_{e}$ the effective thermal conductivity of the porous media given as $\lambda_{e}=\phi \lambda_{f}+(1-\phi) \lambda_{s}$, where $\lambda_{f}$ and $\lambda_{s}$ are thermal conductivities for the fluid and solid phases, respectively. In the final equation $C$ stands for concentration, and $D$ for mass diffusivity. In the momentum equation (2), the additional Brinkman viscous term is included, which express the viscous resistance or viscous drag force exerted by the solid phase on the flowing fluid at their contact surfaces. The density of the fluid depends only on temperature and concentration variations and is described with Boussinesq approximation as:

$$
\rho(T, C)=\rho_{0}\left[1-\beta_{T}\left(T-T_{0}\right)-\beta_{C}\left(C-C_{0}\right)\right],
$$

where $\beta_{T}$ and $\beta_{C}$ are volumetric thermal and concentration expansion coefficients respectively. The equations are coupled through the term $F$ in the momentum equation, which is the density difference function and includes the expression (5) written above.

The boundary conditions for the governing equations can be written as:

- left wall

$$
x=0 ; \quad v_{x}=v_{y}=0, \quad T=1, \quad \frac{\partial C}{\partial x}=0,
$$

- right wall

$$
x=2 ; \quad v_{x}=v_{y}=0, \quad T=0, \quad \frac{\partial C}{\partial x}=0,
$$


- bottom wall

$$
y=0 ; \quad v_{x}=v_{y}=0, \quad \frac{\partial T}{\partial y}=0, \quad C=1,
$$

- top wall

$$
y=2 ; \quad v_{x}=v_{y}=0, \quad \frac{\partial T}{\partial y}=0, \quad C=0 .
$$

The non-slip boundary conditions are prescribed at the impermeable walls of the enclosure. Along the vertical walls the constant temperatures and adiabatic conditions are assumed. The constant species concentrations are assumed along the horizontal walls and zero mass fluxes at the vertical walls of the enclosure.

\section{Numerical procedure}

The governing set of equations (1), (2), (3) and (4) is solved using the extended Boundary Element Method, called also the Boundary Domain Integral Method (BDIM). Since the integral equations are given in terms of variables on the integration boundaries, as well as within the integration domain, the discretization of the solution boundary and domain is required. The whole numerical scheme was already presented at BEM/MRM the details are given in [5], [4]. The main steps to obtain a solution of the described problem with BDIM are:

- introduction of modified velocity in all governing equations: $v_{i}^{\prime}=v_{i} / \phi$,

- introduction of heat diffusivity $a_{T}=\lambda_{e} / \rho c_{f}$ and heat capacity $\sigma=\phi+$ $(1-\phi)(\rho c)_{s} /(\rho c)_{f}$ in the energy equation,

- separation of velocity field into a constant and variable computational parts $v_{i}^{\prime}=\bar{v}_{i}^{\prime}+{\tilde{v^{\prime}}}_{i}$

- separation of material properties (kinematic viscosity, heat diffusivity, species diffusivity) into a constant and variable parts as: $v=\bar{v}+\tilde{v}, a_{T}=$ $\bar{a}_{T}+\tilde{a}_{T}, D=\bar{D}+\tilde{D}$,

- transformation of governing equations with use of velocity-vorticity formulation (with introduction of vorticity vector as a curl of velocity field $\omega^{\prime}=e_{i j} \partial v_{j}^{\prime} / \partial x_{i}$ for the case of planar geometry), which consequently separates the computational scheme into a kinematic and kinetic computational parts,

- derivation of integral equations with use of weighting residual technique or Green fundamental functions, where for the kinematics the elliptic Laplace fundamental solution and for the kinetics the elliptic diffusion-convective fundamental solution are used respectively,

- discretization of solution boundary and domain; all boundary domain integral equations are written for all boundary and domain nodes, where the variation of field functions within each boundary element and internal cell is approximated by the use of appropriate interpolation polynomials,

- solution of coupled matrix system with the use of subdomain technique, where each subdomain consists of four discontinuous 3-node quadratic boundary elements and one 9 -node corner continuous quadratic internal cell. 
The final implicit matrix system for the solution of velocity field and values of vorticity, temperature and concentration is given for the case of planar geometry as:

- Kinematics:

$$
[H]\left\{v_{i}^{\prime}\right\}=e_{i j}\left[H_{t}\right]\left\{v_{j}^{\prime}\right\}-e_{i j}\left[D_{j}\right]\left\{\omega^{\prime}\right\},
$$

- Vorticity kinetics:

$$
\begin{aligned}
{[H]\left\{\omega^{\prime}\right\}=} & \frac{1}{\bar{v}}[G]\left\{v \frac{\partial \omega^{\prime}}{\partial n}-\omega^{\prime} v_{n}^{\prime}+e_{i j} g_{j} F n_{j}+f_{j} n_{j}\right\} \\
& +\frac{1}{\bar{v}}\left[D_{j}\right]\left\{\omega^{\prime} \tilde{v}_{j}^{\prime}-e_{i j} g_{j} F-\tilde{v} \frac{\partial \omega^{\prime}}{\partial x_{j}}-f_{j}\right\} \\
& +\frac{1}{\bar{v} \Delta t}[B]\left\{\frac{\nu \phi}{K} \omega^{\prime}+\omega_{F-1}^{\prime}\right\}
\end{aligned}
$$

- Energy kinetics

$$
\begin{aligned}
{[H]\{T\}=} & \frac{\phi}{\sigma \bar{a}_{p}}[G]\left\{\frac{\sigma a_{p}}{\phi} \frac{\partial T}{\partial n}-T v_{n}^{\prime}\right\}- \\
& -\frac{\phi}{\sigma \bar{a}_{p}}\left[D_{j}\right]\left\{\frac{\sigma \tilde{a}_{p}}{\phi} \frac{\partial T}{\partial x_{j}}-T \tilde{v}_{j}^{\prime}\right\}+\frac{1}{\bar{a}_{p} \Delta t}[B]\{T\}_{F-1},
\end{aligned}
$$

- Species kinetics

$$
\begin{aligned}
{[H]\{C\}=} & \frac{\phi}{\bar{D}}[G]\left\{\frac{D}{\phi} \frac{\partial C}{\partial n}-C v_{n}^{\prime}\right\} \\
& -\frac{\phi}{\bar{D}}\left[D_{j}\right]\left\{\frac{\tilde{D}}{\phi} \frac{\partial C}{\partial x_{j}}-C \tilde{v}_{j}^{\prime}\right\}+\frac{\phi}{\bar{D} \Delta t}[B]\{C\}_{F-1} .
\end{aligned}
$$

The term $f_{j}$ in the vorticity equation describes any contribution arising on account of nonlinear material properties and is given as $f_{j}=\tilde{v}\left(\vec{\nabla} \times \dot{\varepsilon}_{i j}\right)$. The matrix $[H]$, $\left[H_{t}\right],[G],\left[D_{j}\right]$ and $[B]$ are the influence matrices composed of those integrals which took over the individual boundary elements and internal cells.

\section{Results and discussion}

The presented numerical scheme was used to solve a problem of double-diffusive natural convection due to cross temperature and concentration gradients as shown in the chapter 2. The governing parameters that define the fluid flow, heat and solute transfers are:

- aspect ratio $A=L / H$, where $L$ and $H$ are length and height of the enclosure respectively,

- Darcy number $D a=K / H^{2}$,

- porous Rayleigh number $R a=K g_{i} \beta_{T}, \Delta T H / a_{T} v$, where $\Delta T$ is the temperature difference, $a_{T}$ thermal diffusivity and $v$ kinematic viscosity, 
- Lewis number $L e=a_{T} / D$,

- buoyancy coefficient $N=\beta_{C} \Delta C / \beta_{T} \Delta T$, where $\Delta C$ is concentration difference, all other parameters are already described.

In the presented example the results for enclosure of aspect ratio $A=2, R a=100$, $D a=10^{-3}, L e=10$ and different values of buoyancy coefficient are presented. A non-uniform computational mesh $20 \times 10$ was used with a ratio between the longest and shortest elements of $r=6$. Time-steps of $\Delta t=10^{-} 1$ were employed for all cases, and the convergence criterion is determined as $\varepsilon=5 \times 10^{-6}$. In the Figure (2) left the streamlines, isotherms and concentration lines for $N=0$ and right for $N=-1.5$ are shown and in the Figure (3) for $N=-2$ left and $N=-4$ right. For the case $N=0$, where flow is driven by thermal buoyancy force, the flow structure consists of one main circulation occupying the entire enclosure. At the core of the enclosure the concentration gradient reversal is evident, due to strong flow recirculation. From the temperature filed is evident that the rate of heat transfer is high at the bottom of the hot wall and upper part of the cold wall. The rate of heat transfer decreases along the hot wall. With increase of buoyancy coefficient $N$ in negative sense the strength of the flow circulation decreases and starts to bifurcate into two weak circulations. Physical explanation for the cases when $|N|>0$ is that heavy fluid particles (high concentration) that advected to the upper part of the enclosure reach the other end and form recirculation. As $N$ decreases the
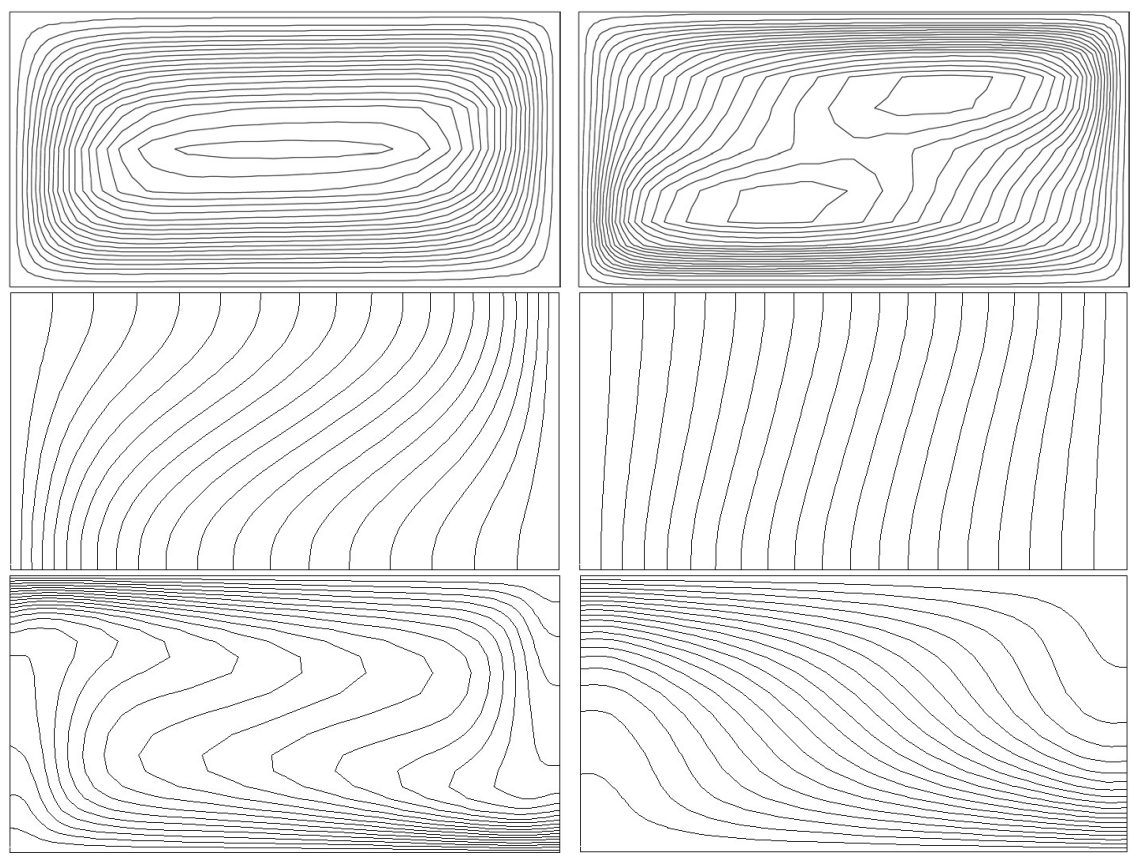

Figure 2: Streamlines, isotherms and isoconcentrations for $A=2, R a=100$, $D a=10^{-3}, L e=10$ and $N=0$ (left) and $N=-1.5$ (right). 

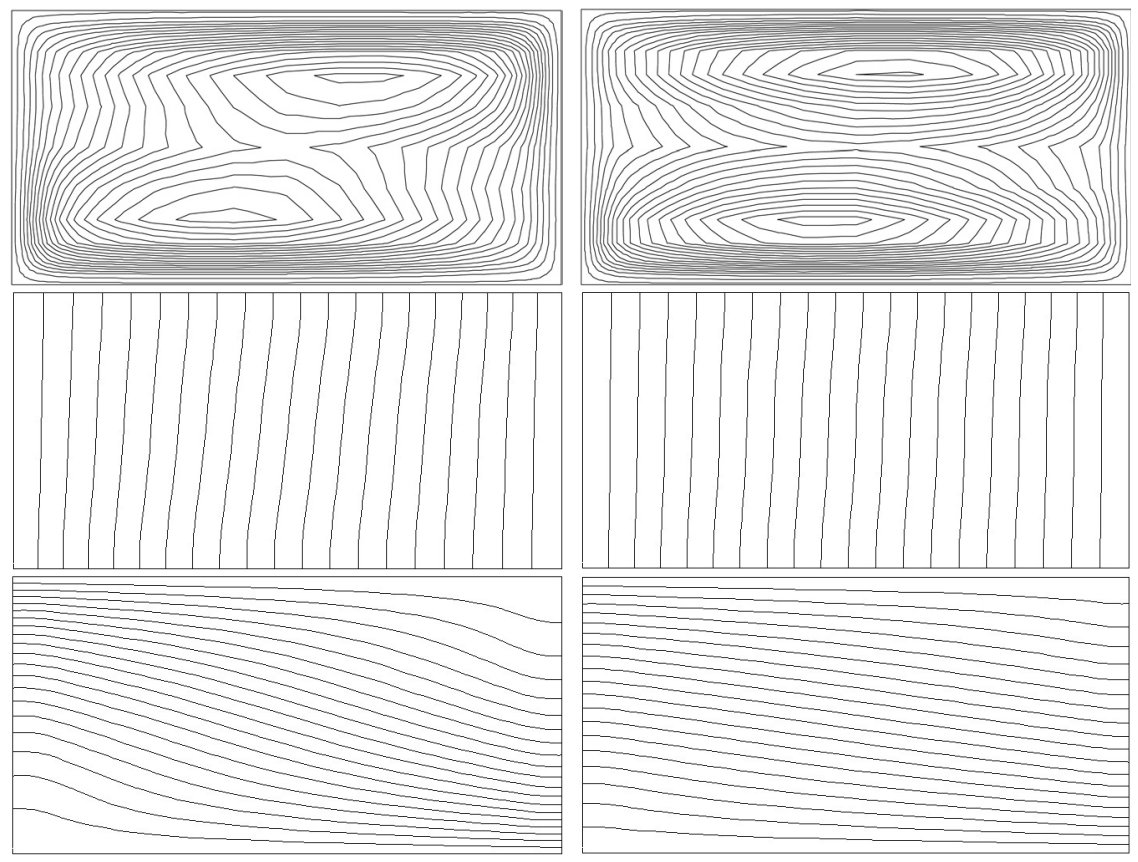

Figure 3: Streamlines, isotherms and isoconcentrations for $A=2, R a=100$, $D a=10^{-3}, L e=10$ and $N=-2$ (left) in $N=-4$ (right).

heavy particles sink before they arrive at the other end of the enclosure forming two circulations. With increase of $N$ also the concentration reversal and isotherms distortion diminish. For $N=-4$ heat transfer takes place mainly by conduction, which is evident from the isotherm distribution. The sharp corners in the streamline presentations in Figure (3) are due to small grid density $(20 \times 10$ subdomains). For better graphical presentations denser grids have to be used. Table (1) shows results for the values of Nusselt and Sherwood numbers for the examples shown in Figures 2 and 3. As already stated from the graphical presentations, the heat is for small values of $N$ transfered by conduction, while the solute is transported by the convection mechanism. The results are compared to the reference [3], where the same problem is solved for planar and spatial geometry with use of finite difference method (FDM). The agreement between the results is good, which proves the accuracy of mathematical model and BDIM.

\section{Conclusion}

In the paper the results for double-diffusive natural convection in a rectangular enclosure subjected to cross gradients of temperature and concentration, obtained with use of extended Boundary Element Method are presented. The results show that the competition between solutal and thermal force produce complex flow pat- 
Table 1: $N u$ and $S h$ values for different $N$ and $A=2, R a=100, L e=10$, $D a=10^{-3}$.

\begin{tabular}{llcccc}
\hline$N$ & & -4 & -2 & -1.5 & -1 \\
\hline$N u$ & Present results & $\mathbf{0 . 4 9}$ & $\mathbf{0 . 5 0}$ & $\mathbf{0 . 5 0}$ & $\mathbf{0 . 5 2}$ \\
& Ref. [3] & - & 0.5 & 0.5 & 0.55 \\
\multirow{2}{*}{$S h$} & Present results & $\mathbf{1 . 0 4}$ & $\mathbf{1 . 1 7}$ & $\mathbf{1 . 3 0}$ & $\mathbf{1 . 7 1}$ \\
& Ref. [3] & - & 1.2 & 1.3 & 1.9 \\
\hline
\end{tabular}

terns. The dependency of buoyancy coefficient on the convective flow in the enclosure is investigated and it is stated that with increase of buoyancy coefficient $N$ in negative sense the convective motion slows down and approaches the pure diffusion limit. The results are compared to a published study, where the FDM was used to solve the same problem. Very good agreement between the results is stated, which indicates that the BDIM could be efficiently used for solving the doublediffusive natural convection problem in porous media.

\section{References}

[1] Mohamad, A.A. \& Bennacer, R., Natural convection in a confined saturated porous medium with horizontal tempertaure and vertical solutal gradients. Int J Therm Sci, 40, pp. 82-93, 2001.

[2] Kalla, L., Vasseur, P., Beji, H. \& Duval, R., Double diffusive convection within a horizontal porous layer salted from the bottom and heated horizontally. Int Comm Heat Mass Transfer, 28, pp. 1-10, 2001.

[3] Mohamad, A.A. \& Bennacer, R., Double diffusion, natural convection in an enclosure filled with saturated porous medium subjected to cross gradients; stably stratified fluid. Int J Heat Mass Transfer, 45, pp. 3725-3740, 2002.

[4] Kramer, J., Jecl, R. \& Škerget, L., Boundary element method for double diffusive natural convection in horizontal porous layer. Boundary elements and other mesh reduction methods XXIX, 2007.

[5] Škerget, L., Hriberšek, M. \& Kuhn, G., Computational fluid dynamics by boundary-domain integral method. Int J Numer Meth Engng, 46, p. 1291.1311, 1999. 